\title{
Adult systemic Epstein-Barr virus-positive T-cell lymphoproliferative disease: A case report
}

\author{
YOUPING WANG ${ }^{1}$, XINYUE LIU $^{2}$ and YAN CHEN ${ }^{2}$ \\ ${ }^{1}$ Department of Oncology, Second Affiliated Hospital, School of Medicine, Zhejiang University, Hangzhou, Zhejiang 310009; \\ ${ }^{2}$ Department of Hematology, The Union Hospital, Tongji Medical College, Huazhong University of Science and Technology, \\ Wuhan, Hubei 430022, P.R. China
}

Received July 16, 2014; Accepted May 21, 2015

DOI: $10.3892 /$ etm.2015.2601

\begin{abstract}
Systemic Epstein-Barr virus (EBV)-positive T-cell lymphoproliferative disease $\left(\mathrm{EBV}^{+} \mathrm{T}\right.$-LPD) occurs mainly in Asia and South America and is extremely rare in adults. The disease is characterized by a clonal proliferation of EBV-infected T cells with a cytotoxic immunophenotype and is associated with a poor clinical outcome and can be life-threatening. The majority of the patients have evidence of systemic disease, often with lymph node, liver and spleen involvement. The present study describes a case of adult systemic $\mathrm{EBV}^{+} \mathrm{T}-\mathrm{LPD}$ with high fever, systemic lymphadenopathy, hepatosplenomegaly, nose-pharynx neoplasm, pancytopenia, EB virus infection and proliferative bone marrow, with the aim of improving the understanding of the condition.
\end{abstract}

\section{Introduction}

The oncogenic potential of Epstein-Barr virus (EBV) is associated with its ability to infect and transform $\mathrm{T}$ lymphocytes into continuously proliferating lymphoblastoid cells. The virus has also been implicated in the development of T-cell lymphoproliferative diseases (T-LPDs). EBV-positive T-LPD $\left(\mathrm{EBV}^{+} \mathrm{T}-\mathrm{LPD}\right)$ includes the polyclonal, oligoclonal and monoclonal proliferation of cytotoxic T cells (1). This disease is rare, with high rates of morbidity and mortality and is more prevalent in Eastern Asian countries (2). The disease is associated with a poor prognosis, a progressive clinical manifestation, diverse pathological types and cell clones and numerous stages of development, which are different from either the benign

Correspondence to: Professor Xinyue Liu and Dr Yan Chen, Department of Hematology, The Union Hospital, Tongji Medical College, Huazhong University of Science and Technology, 1277 Jiefang Avenue, Wuhan, Hubei 430022, P.R. China

E-mail:1xy87306548@sina.com

E-mail: yanchen981@163.com

Key words: Epstein-Barr virus, lymphoproliferative disease, natural killer/T-cell lymphoma, infectious mononucleosis lesions of infectious mononucleosis or typical lymphoma lesions. To avoid over- and under-diagnosis, the possibility of a single disease having different stages of development, as well as the consideration that the combination of clinical and laboratory findings with the pathological and immunohistochemical characteristics can be beneficial in reaching the correct diagnosis, should be taken into account. The present study describes a case of adult systemic EBV ${ }^{+}$T-LPD $\left(\mathrm{ASEBV}^{+} \mathrm{T}-\mathrm{LPD}\right)$.

\section{Case report}

A 21-year old female patient with a 20-day history of high fever, fatigue and yellow sclerae was admitted to The Union Hospital, Tongji Medical College (Wuhan, China) on September 1,2010. The patient had no other medical history of note. Written informed consent was obtained from the patient for the present report.

The physical examinations that were performed on admission showed yellowing of the skin and sclerae, a soft lymph node without tenderness in the left of the neck and the right side of the groin, and grade III-bilateral tonsillar enlargement with pus emboli. The liver could not be located through touch, but the spleen was felt $3 \mathrm{~cm}$ below the rib. The results of the laboratory tests performed are shown in Table I. The cytological examination of the bone marrow exhibited karyocytic hyperplasia (Fig. 1). Granulocytes accounted for $43.0 \%$ of the cell population, erythrocytes for $34.5 \%$, lymphocytes for $19.0 \%$ and heterotypic lymphocytes for $3.5 \%$. Flow cytometric analysis of the bone marrow revealed a reduction in the cluster of differentiation $\mathrm{CD}^{+} \mathrm{CD}^{+} / \mathrm{CD}^{+}{ }^{+} \mathrm{CD} 8^{+}$ratio, with a value of 0.57. Lymph node immunophenotyping showed that lymphocytes accounted for $80 \%$ of the karyocytes; of the lymphocytes, $\sim 40 \%$ were B lymphocytes and $\sim 58.75 \%$ were $\mathrm{CD}^{+} 6^{+}$cells. The $\mathrm{CD} 6^{+}$cells were additionally found to be positive for T-antigens, such as CD2, CD3, CD5, T cell receptor (TCR) $\alpha / \beta$ and human leukocyte antigen (HLA)-DR, suggesting the existence of abnormal natural killer (NK)/T cells. Pathological examination of the cervical lymph nodes revealed that the basic structural features, including lymph sinus and reactive lymphoid follicles, were present. Immunohistochemistry showed that infiltrated cells were T-cell-restricted intracellular antigen 1 (TIA-1) (+), Ki67 (+) (20\% only), CD4 (-), CD8 (+), CD2 (-), 
CD5 (+), anaplastic lymphoma kinase (ALK) (-), CD20 (+), CD3 (+), CD21 (+) (follicular dendritic cells only), CD30 (-) and paired box protein Pax-5 (-) (Fig. 2). The pathological diagnosis was atypical T-cell hyperplasia of the lymph node.

The patient was administered liver protection treatment and supporting therapy, which led to the gradual recovery of liver function; however, she still presented with fever and superficial lymphadenopathy, resulting in the suspicion of lymphoma. On September 18, a neoplasm was found in the region of the pharynx lying above the soft palate of the patient, and she was subjected to tonsillectomy and neoplasm biopsy. Pathological examination of the nasopharynx and bilateral tonsil showed chronic inflammatory changes (Fig. 3). Immunohistochemistry of the nasopharynx showed that the infiltrated cells were diffusely positive for CD20, CD3 and Ki67 (outside the germinal center) but negative for CD15. A small number of cells were positive for CD30.

Due to the atypical nature of the pathological characteristics, the patient visited another hospital (Beijing Friendship Hospital, Capital University of Medical Sciences, Beijing, China) and was examined for other cervical lymph node markers, giving the following results: Myeloperoxidase (MPO) (-), CD34 (-) and EBV-encoded RNA (EBER) (+) (Fig. 4). The cells exhibited marked proliferation and morphologically resembled lymphoma cells. T-cell markers were expressed in the proliferating cells, with the exception of $\mathrm{CD} 2$, indicating tumorigenesis; however, the absence of tumor-related immune markers did not favor a diagnosis of lymphoma. EBV-infected lymph node inflammation was pathologically diagnosed. On September 28 , the body temperature of the patient continued to rise (maximum, $40^{\circ} \mathrm{C}$ ), and numerous pus emboli had adhered to the posterior wall of the nasopharynx. Magnetic resonance imaging (MRI) of the nasopharynx revealed a thickened mucous membrane but no signals indicative of an abnormal mass. The antigen receptor gene rearrangement test and the immunoglobulin heavy- and light-chain gene rearrangement studies by polymerase chain reaction failed to demonstrate conclusive evidence of a clonal B- or T-cell population. The conventional chromosomal study revealed a normal karyotype. Given the changes in the condition of the patient, $\mathrm{ASEBV}^{+} \mathrm{T}$-LPD was clinically diagnosed. During hospitalization, the patient was given anti-infection treatment (cefepime, fluconazole, etc.); her body temperature returned to the normal level, the jaundice disappeared and no systemic superficial lymphadenopathy or hepatosplenomegaly was detected. The patient was discharged on October 15, 2010 following the improvement in her condition. During a two-year follow-up period the patient had no fever or enlarged superficial lymph nodes, and the peripheral blood examination and liver and kidney function tests were normal.

\section{Discussion}

The incidence of EBV infection in the Chinese population is $90 \%$, and $\mathrm{EBV}$ is the common pathogenic factor of numerous diseases, including infectious mononucleosis (IM), Burkitt lymphoma and NK/T-cell lymphoma (3,4). In addition, EBV infection is closely associated with certain LPDs that are in a stage of development between tumor and non-cancer. In 2008, the World Health Organization classified $\mathrm{EBV}^{+} \mathrm{T}$-LPD (1)
Table I. Laboratory tests.

Test

Result

$\begin{array}{lc}\text { Routine blood } & 3.24 \times 10^{9} \\ \text { WBC }(\mathrm{g} / \mathrm{l}) & 98 \\ \mathrm{Hb}(\mathrm{g} / \mathrm{l}) & 1.85 \times 10^{11} \\ \text { PLT }(\mathrm{g} / \mathrm{l}) & \\ \text { Liver } / \text { kidney function } & 417 \\ \mathrm{TBA}(\mu \mathrm{mol} / \mathrm{l}) & 231 \\ \mathrm{~TB}(\mu \mathrm{mol} / \mathrm{l}) & 881 \\ \mathrm{DB}(\mu \mathrm{mol} / \mathrm{l}) & 417 \\ \mathrm{ALT}(\mathrm{U} / \mathrm{l}) & 231 \\ \mathrm{AST}(\mathrm{U} / \mathrm{l}) & 881 \\ \text { ALP }(\mathrm{U} / \mathrm{l}) & 29.4 \\ \text { ALB }(\mathrm{g} / \mathrm{l}) & 5.7 \\ \mathrm{~K}^{+}(\mathrm{mmol} / \mathrm{l}) & \end{array}$

Imaging

Bronchitis, bilateral axillary lymph node enlargement, obvious hepatosplenomegaly

Others

Serum EBVCA-IgM
Bacterial/viral/tumor antigens
Heterophil agglutination

Positive

Negative

Negative

WBC, white blood cells; Hb, hemoglobin; PLT, platelets; TBA, thiobarbituric acid; TB, total bilirubin; $\mathrm{DB}$, direct bilirubin; ALT, alanine transaminase; AST, aspartate aminotransferase; ALP, alkaline phosphatase; ALB, albumin; EBVCA-IgM, Epstein-Barr virus capsid antigen-immunoglobulin $\mathrm{M}$.

into childhood systemic $\mathrm{EBV}^{+} \mathrm{T}-\mathrm{LPD}\left(\mathrm{CSEBV}^{+} \mathrm{T}-\mathrm{LPD}\right)$ and ASEBV $^{+}$T-LPD. ASEBV ${ }^{+}$T-LPD is a rare disease characterized by EBV-infected T-cell proliferation with a cytotoxic phenotype. It has been suggested that, in the early stages of $\mathrm{CSEBV}^{+} \mathrm{T}$-LPD, EBV-infected cells exhibit poly- or oligoclonal proliferation, which often progresses to monoclonal proliferation in the later disease stages (2), suggesting that $\mathrm{CSEBV}^{+} \mathrm{T}-\mathrm{LPD}$ is essentially a disease spectrum that incorporates different stages of development, ranging from benign to malignant proliferation (5). The case reported in the present study was clinically characterized by a subacute onset, moderate-to-severe fever, systemic lymphadenopathy, hepatosplenomegaly, swollen tonsils, bronchitis, jaundice, pancytopenia, EBV infection and bone marrow proliferation. Pathological examination of the lymph nodes revealed expansion of the interfollicular area, which was diffusely infiltrated by a polymorphous infiltrate of small-to-medium-sized lymphocytes, plasma cells and immunoblasts. In terms of immunohistochemistry, the infiltrated cells had a strong, diffuse positivity for CD3, CD8, CD5, TIA and EBER, and CD20 staining was present in admixed normal-appearing B-lymphocytes. The infiltrated cells were negative for CD2, CD4, CD30, CD34, CD117, MPO, ALK and latent membrane protein 1 . Overall, the pathological and immunohistochemical studies of the cervical lymph nodes were indicative of early-stage disease, which is coincident with the A1 


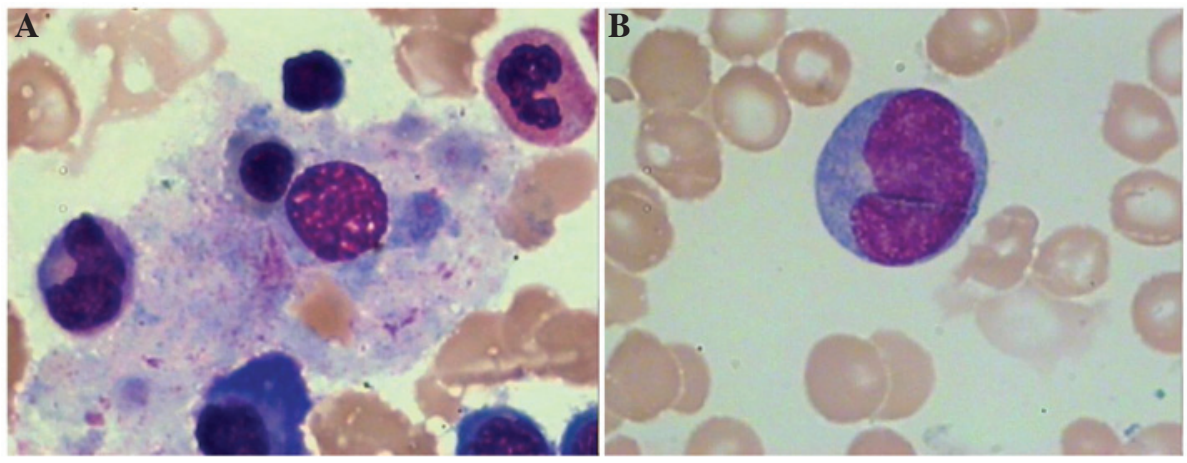

Figure 1. Cytological examination of the bone marrow. (A) Ingestion of an erythrocyte by a macrophage in the aspirated bone marrow smear (HE staining; magnification, $\mathrm{x} 400$ ); (B) Morphological changes of heterotypic lymphocytes within the bone marrow (HE staining; magnification, $\mathrm{x} 400$ ). HE, hematoxylin and eosin.

A

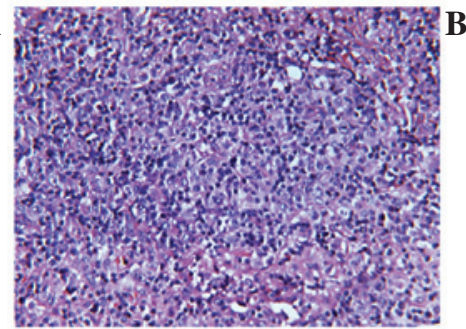

D

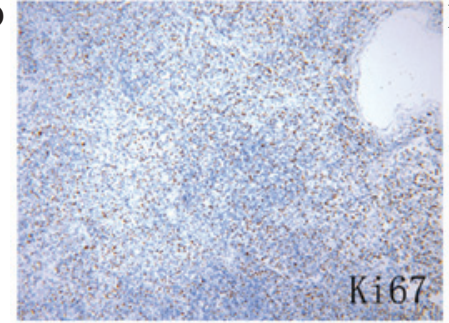

\section{(1)}
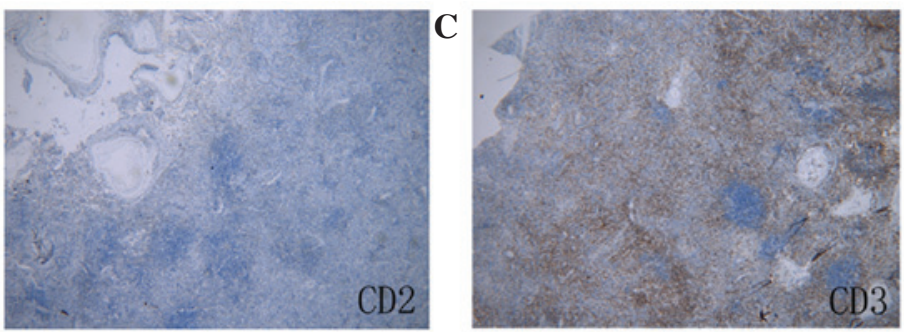

E

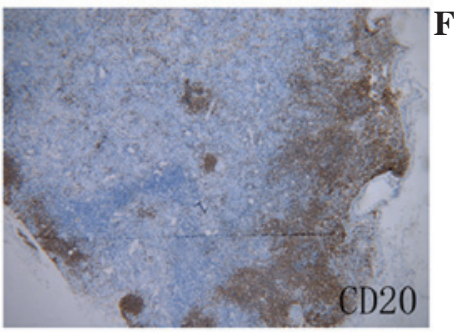

Figure 2. (A) Biopsies of the cervical lymph nodes revealed that the paracortical area was diffusely infiltrated by a polymorphous infiltrate of small-to-medium-to-large-sized (transformed) lymphocytes, immunoblasts and mature plasma cells. These cells were chromatin-rich, increased in size and had an enlarged nucleus (HE staining; magnification, x400). The infiltrated cells were (B) negative for CD2 but (C-F) positive for (C) CD3, (D) Ki67, (E) CD20 and (F) CD5 (HE staining; magnification, $\mathrm{x} 40$ ). HE, hematoxylin and eosin; CD, cluster of differentiation.

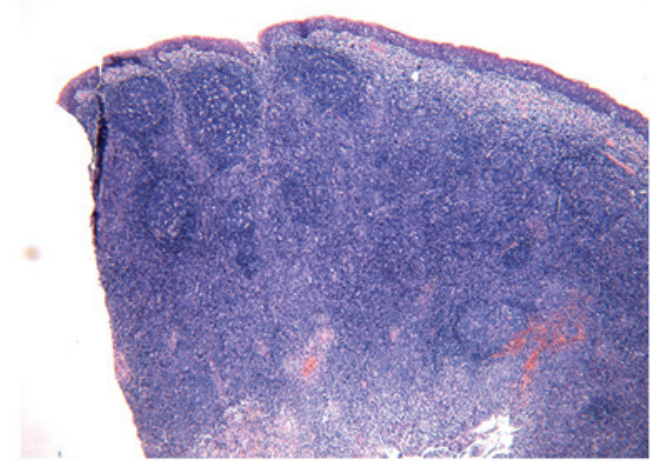

Figure 3. Sample from a bilateral tonsil biopsy showing lymphocyte infiltration and proliferation, lymphoid follicle formation and irregular expansion (hematoxylin and eosin staining; magnification, $\mathrm{x} 40$ ).

category from the classification of $\mathrm{EBV}^{+} \mathrm{NK} / \mathrm{T}-\mathrm{LPD}$ proposed by Ohshima et al (2).

In the present case, the patient exhibited a grade III-bilateral tonsil enlargement with pus emboli; bone marrow smear showed heterotypic lymphocytes accounting for $3.5 \%$ of the

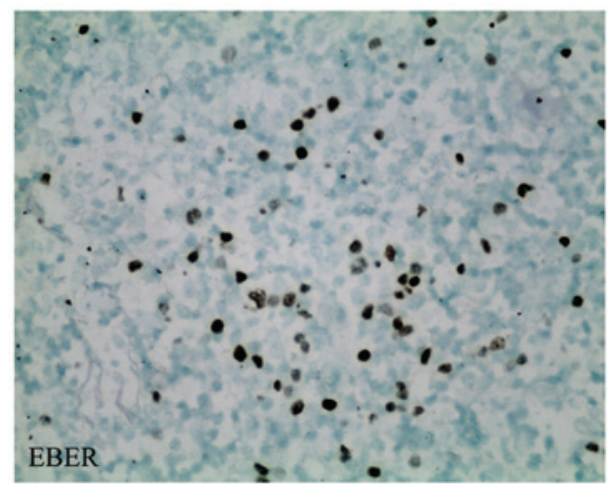

Figure 4. Infiltrated cells in the paracortical area stained positive for Epstein-Barr virus latent membrane protein antigen using in situ hybridization (magnification, x400). EBER, Epstein-Barr virus-encoded RNA.

cell population. Immunophenotypic analysis of the lymph nodes revealed that the $\mathrm{CD}_{5} 6^{+}$cells accounted for $58.75 \%$ of the lymphocytes and that T-antigens, such as CD2, CD3, CD5, TCR $\alpha / \beta$ and HLA-DR, were also simultaneously expressed in certain $\mathrm{CD}^{2} 6^{+}$cells, which could be considered as abnormal 
$\mathrm{NK} / \mathrm{T}$ cells. As the disease progressed, a nasopharyngeal neoplasm was detected, which, based on the clinical features and immunohistochemical analysis, could have led to a misdiagnosis of NK/T-cell lymphoma. NK/T-cell lymphoma characteristically arises in the nasal cavity or surrounding structures and manifests as a destructive midline facial lesion with tumor cells expressing CD2, CD56, cytotoxic granule proteins, cytoplasmic CD3 and TIA-1, but not CD3 $(6,7)$. Although the immunophenotypic analysis of the cervical lymph nodes in the present case revealed abnormal NK/T-cell proliferation, the tonsil and nasopharyngeal neoplasm biopsy indicated inflammatory changes, and the nasopharyngeal MRI scan showed a thickened mucous membrane but no signals indicative of an abnormal mass. The immunophenotyping of the cervical lymph nodes showed negativity for CD2 and positivity for CD3. A number of the present findings indicate the possibility of progression to NK/T-cell lymphoma, and due caution should therefore be taken. These findings included the fact that i) the infiltrating cells were small-to-medium-sized lymphocytes with large cells exhibiting different degrees of atypia scattered among them; ii) CD8 expression was present in the majority of the cells; iii) there was diffuse CD3-positivity; and iv) the Ki67-positivity was $<30 \%$ (5).

The differential diagnosis of $\mathrm{ASEBV}^{+} \mathrm{T}-\mathrm{LPD}$ versus IM was raised due to the atypical pathology of cervical lymph nodes. The clinical and pathological diagnosis of IM was described by Chen et al (8) and Zhou (9). The characteristic pathological changes described included expansion of the paracortical area and morphological changes in the B-cell differentiation spectrum (lymphoblasts, immunoblasts, plasmacytoid cells, mature plasma cells), with CD3-positivity and varying degrees of scattered CD20 and CD30-positivity; however, in the present case the lesion did not contain CD30 (+) cells and the pathological examination of the lymph nodes did not demonstrate the typical pathological changes of IM, i.e. B-cell differentiation spectrum changes. To avoid over- and under-diagnosis, it is necessary to consider that a single disease has different stages of development, and the combination of clinical and laboratory findings with pathological and immunohistochemical features is beneficial for the correct diagnosis.

\section{Acknowledgements}

The authors would like to thank Department of Pathology, Peking University Health Science Center for the pathological images.

\section{References}

1. Quintanilla-Martinez L, Kimura H and Jaffe ES. Epstein-Barr virus (EBV) positive T-cell lymphoproliferative diseases of childhood. In: Swerdlow SH, Campo E, Harris NL, Jaffe ES, Pileri SA, Stein H, Thiele J and Vardiman JW (eds.): WHO Classification of Tumours of Haematopoietic and Lymphoid Tissues. Vol. 2. 4th edition. IARC Press, Lyon, pp278-280, 2008.

2. Ohshima K, Kimura H, Yoshino T, Kim CW, Ko YH, Lee SS, Peh SC and Chan JK; CAEBV Study Group: Proposed categorization of pathological states of EBV-associated T/natural killer-cell lymphoproliferative disorder (LPD) in children and young adults: Overlap with chronic active EBV infection and infantile fulminant. EBV T-LPD. Pathol Int 58: 209-217, 2008

3. Lai HH and Ma L: Research progress of EB virus related malignancies. Zhongguo Xiao Er Yu Xue Ye Za Zhi 18: 245-249, 2013 (In Chinese).

4. Quintanilla-Martinez L, Kumar S, Fend F, Reyes E, Teruya-Feldstein J, Kingma DW, Sorbara L, Raffeld M, Straus SE and Jaffe ES: Fulminant EBV(+) T-cell lymphoproliferative disorder following acute/chronic EBV infection: A distinct clinicopathologic syndrome. Blood 96: 443-451, 2000.

5. Jin Y, Zhou XG, He LJ, Xie JL, Zheng YY, Zhang YN and Zhang SH: Clinicopathologic features of systemic EBV-positive T-cell lymphoproliferative disease of childhood. Zhonghua Bing Li Xue Za Zhi 38: 600-608, 2009 (In Chinese)

6. Yachie A, Kanegane H and Kasahara Y: Epstein-Barr virus-associated T-/natural killer cell lymphoproliferative diseases. Semin Hematol 40: 124-132, 2003.

7. Li CC, Tien HF, Tang JL, Yao M, Chen YC, Su IJ, Hsu SM and Hong RL: Treatment outcome and pattern of failure in 77 patients with sinonasal natural killer/T-cell or T-cell lymphoma. Cancer 100: 366-375, 2004

8. Chen YZ, Zhou XG, Jin Y, Zheng YY, Chen G and Shi Y: Study of clinical and morphological features, immunophenotype and Epstein-Bar virus infection in situ of infectious mononucleosis. Zhonghua Bing Li Xue Za Zhi 37: 440-444, 2008 (In Chinese).

9. Zhou XG: Increasing recognition of T zone lymphoproliferative disorders. Zhonghua Bing Li Xue Za Zhi 36: 73-75, 2007 (In Chinese). 